\title{
Activation and Evasion of RLR Signaling by DNA Virus Infection
}

\author{
Junli Jia ${ }^{1 \dagger}$, Jiangan $\mathrm{Fu}^{2+}$ and Huamin Tang ${ }^{1,3,4 *}$ \\ ${ }^{1}$ Department of Immunology, Nanjing Medical University, Nanjing, China, ${ }^{2}$ Genor Biopharma Co., Ltd., Shanghai, China, \\ ${ }^{3}$ Laboratory Center for Basic Medical Sciences, Nanjing Medical University, Nanjing, China, ${ }^{4}$ Key Laboratory of Antibody \\ Technique of Ministry of Health, Nanjing Medical University, Nanjing, China
}

\section{OPEN ACCESS}

Edited by:

Chunfu Zheng,

University of Calgary, Canada

Reviewed by:

Cao-Qi Lei,

Wuhan University, China

Erguang Li,

Nanjing University, China

*Correspondence:

Huamin Tang

htang@njmu.edu.cn

tThese authors have contributed equally to this work

Specialty section: This article was submitted to

Microbial Immunology, a section of the journal

Frontiers in Microbiology

Received: 29 October 2021 Accepted: 02 December 2021 Published: 20 December 2021

Citation:

Jia J, Fu J and Tang H (2021) Activation and Evasion of RLR

Signaling by DNA Virus Infection.

Front. Microbiol. 12:804511. doi: 10.3389/fmicb.2021.804511
Antiviral innate immune response triggered by nucleic acid recognition plays an extremely important role in controlling viral infections. The initiation of antiviral immune response against RNA viruses through ligand recognition of retinoic acid-inducible gene I (RIG-I)-like receptors (RLRs) was extensively studied. RLR's role in DNA virus infection, which is less known, is increasing attention. Here, we review the research progress of the ligand recognition of RLRs during the DNA virus infection process and the viral evasion mechanism from host immune responses.

Keywords: RIG-I-like receptor, RNAPIII, MAVS, MDA5, LPG2

\section{INTRODUCTION}

The innate immune response is the first line of host defense against microbial infection (Zhao and Karijolich, 2019). Pattern-recognition receptors (PRRs) detect pathogen-associated molecular patterns (PAMPs) structures, most of which, if not all, are crucial for their life cycle. Pathogenspecific sequences of DNA and RNA present in viral, bacterial, and eukaryotic genomes are one of the important categories of PAMPs detected by host PRRs (Goubau et al., 2013; Liu and Gack, 2020). A set of nucleic-acid-detecting PRRs have been identified, including Toll-like receptors (TLRs), RIG-I-like receptors, NOD-like receptors (NLRs), and other cytosolic and nucleic sensors. Some are located in intracellular compartments, and others are in the cytoplasm, nucleus, or cellular membrane (Ablasser and Sun, 2019). RLRs recognize RNA virus genomes and/or their products, which plays an important role in initiating the antiviral innate immune response against various RNA viruses (Yoneyama et al., 2004; Rehwinkel and Gack, 2020). Interestingly, activation and downstream signal transduction of RLRs have also been recently observed during several DNA viruses' infections (Andrejeva et al., 2004; Gack et al., 2009; Ban et al., 2018; Koliopoulos et al., 2018; Zhang et al., 2020; Jiang et al., 2021).

\section{RLR DISCOVERY AND THEIR STRUCTURE}

Three members of the RLR family, RIG-I (also named as DDX58), MDA5 (melanomadifferentiation-associated gene 5), and LGP2 (laboratory of genetics and physiology 2), have been identified to be expressed in most types of cells and tissues (Yoneyama et al., 2005).

RIG-I was originally identified as a retinoic acid-treated cell induction gene from acute promyelocytic leukemia (Liu et al., 2000). Subsequently, porcine RIG-I homologs were identified 
as porcine reproductive and respiratory syndrome virus-induced genes, suggesting a possible association between RIG-I and viral infection (Zhang et al., 2000). Later studies have shown that RIG-I interacts with intermediate proteins called MAVS (mitochondrial antiviral signaling; IPS-1/VISA/Cardif) during downstream signal transduction, ultimately leading to activation IRF3/7 (interferon regulatory factors 3 and 7) and/or NF-кB transcription factors (Kawai et al., 2005; Meylan et al., 2005; Seth et al., 2005; Xu et al., 2005), which results in the production of type I interferons (IFNs) and other proinflammatory cytokines (Wu et al., 2013; Peisley et al., 2014; Onomoto et al., 2021).

MDA5 is the closest relative of RIG-I, exhibiting 23 and $35 \%$ sequence similarities in the $\mathrm{N}$-terminal and C-terminal domains, respectively (Kang et al., 2002). MDA5 was identified as an upregulated gene in a human melanoma cell line, HO- 1 cells, after combined treatment with IFN- $\beta$ and MEZ (an anti-leukemic PKC activating compound that induces terminal differentiation). Thus, initially, it was thought to be a type I interferon-responsive apoptosis-inducing gene (Kang et al., 2004). Later, MDA5 was found as a binding target for $\mathrm{V}$ proteins of paramyxoviruses (simian virus 5, human parainfluenza virus 2, mumps virus, Sendai virus, and hendra virus). Since V proteins inhibit the dsRNA-induced activation of the IFN-induced genes, this indicates MDA5 has a role in antiviral immune responses (Andrejeva et al., 2004; Rodriguez and Horvath, 2014).

LGP2 has 31 and $41 \%$ sequence similarities with the helicase domains of RIG-I and MDA5, respectively (Rothenfusser et al., 2005; Yoneyama et al., 2005; Komuro and Horvath, 2006). Expression of LGP2 inhibits the signaling to IFN-stimulated regulatory element (ISRE)- and NF- $\mathrm{B}$-dependent pathways induced by infection with Sendai virus and Newcastle disease virus (Rothenfusser et al., 2005). LGP2 can compete with the kinase IKK- $i$ for a common interaction site on MAVS, inhibiting NF- $\kappa$ B-dependent signaling pathway activation. Thus, LGP2 is proposed as a regulatory element of intracellular antiviral signaling (Komuro and Horvath, 2006).

All three RLRs are members of the nucleic acid-dependent NTPases superfamily 2 (SF2) and share two structure similarities, a central helicase domain (responsible for intrinsic dsRNA binding and ATP hydrolysis functions) and a carboxy-terminal domain (CTD; responsible for binding to RNA termini) which are involved in the detection of immunostimulatory RNAs (Luo et al., 2011, 2013). Only RIG-I and MDA5 have two caspase activation and recruitment domains (CARDs) in their $\mathrm{N}$-terminal region, which are responsible for downstream signal transduction. In uninfected cells, RIG-I CARDs are found in a resting state by folding with the helical insertion domain in its helicase domains (Kowalinski et al., 2011), whereas MDA5 activity is repressed by phosphorylation (Wies et al., 2013). After recognition and binding to immunostimulatory RNA, RLRs hydrolyze ATP and change their conformation, resulting in the release of CARDs for MAVS interactions and signal transduction (Myong et al., 2009; Peisley et al., 2013; Devarkar et al., 2018). LGP2 lacks the CARDs and is commonly known to regulate RIGI and MDA5 (Kato et al., 2008; Schlee et al., 2009; Schmidt et al., 2009; Duic et al., 2020).

\section{LIGAND RECOGNITION BY RLRS}

Since host RNAs are present together with pathogen RNAs in the cytosol of infected cells, RLRs need to discriminate immunostimulatory pathogen RNAs from physiologically normal RNAs. Cellular mRNAs, often single-stranded and 5 -triphosphorylated, acquire a cap structure involving 2'-Omethylation (cap 1 or cap 2) in the cell nucleus following transcription (Zuest et al., 2011; Schuberth-Wagner et al., 2015). Typical 28S and $18 \mathrm{~S}$ ribosomal RNAs (rRNAs) and tRNAs have only a single phosphate at the $5^{\prime}$ end. Although $5 S$ rRNA contains a $5^{\prime}$-PPP moiety, it is methylated and complexed with proteins (Chiang J.J. et al., 2018). These host RNAs normally do not have features required for RLR recognition. RNA structures recognized by RIG-I are relatively well elucidated. These RNAs are double-stranded, with $5^{\prime}$-triphosphate or diphosphate, and either have an incompletely methylated cap structure (cap 0 ) (Decroly et al., 2012) or are bearing a protein covalently attached to the $5^{\prime}$ end (such as VPg) (Cardenas et al., 2006). It has also been reported that single-strand viral genomes possessing $5^{\prime}$-phosphorylated feature (without cap structure) can trigger RIG-I/MAVS signaling (Hornung et al., 2006; Pichlmair et al., 2006). Ren et al. (2019) recently reported that $5^{\prime}$-monophosphate RNAs block RIG-I upon binding to the RIG-I CTD domain.

The sensing and activation of RIG-I like receptors are not well understood, although much work has been done to elucidate RNA-triggered MDA5 activation. MDA5 forms filaments with a ring-like conformation around dsRNA in a sequence-nonspecific manner (Berke and Modis, 2012; Peisley et al., 2012). In addition, long poly I: C molecules preferentially trigger MDA5, whereas short poly I: C ones activate RIG (Kato et al., 2008; Berke and Modis, 2012; Wu et al., 2013). Therefore, now it is commonly accepted that MDA5 detects long dsRNA and their secondary structure.

The mechanisms by which LGP2 regulates RIG-I/MDA5mediated signaling remain to be further investigated (Li et al., 2009; Uchikawa et al., 2016). Both positive and negative regulatory function of LGP2 was reported (Uchikawa et al., 2016). In vitro studies showed that LGP2 could affect dsRNA recognition and MAVS interaction of RIG-I and MDA5 and compete with IKK- $i$ for recruitment to MAVS (Komuro and Horvath, 2006; Saito et al., 2007). Lgp $2^{-/-}$mice and mice harboring a point mutation in the LGP2 helicase domain (K30A) showed that LGP2 functions as a positive regulatory factor and enhances RIGI- and MDA5-mediated antiviral activity (Satoh et al., 2010). Furthermore, by using biochemical and biophysical approaches, LGP2 was found to be incorporated into the fibers of MDA5, promoting the exposure of its CARDs, resulting in a change to active conformation and signaling activity (Kato et al., 2006; Bruns et al., 2014; Duic et al., 2020).

\section{MODULATION OF RLRs ACTIVATION}

Innate immune signaling is tightly controlled to prevent excessive inflammatory responses. Post-translational modifications (PTMs) play an important role during this process, including 
phosphorylation, ubiquitination, ISGylation, acetylation, and other less well-studied modifications (Du et al., 2018). Among them, ubiquitination modification is extensively investigated.

TRIM25 is the first E3 ligase identified for CARD-mediated signaling activation of RLG-I. Subsequent studies also showed that modification of RIG-I by TRIM25 is also influenced by several other host factors (Gack et al., 2007). The mitochondrialtargeting chaperone $14-3-3 \varepsilon$ forms a complex with RIG-I and TRIM25 and regulates their transition from the cytoplasm to the mitochondrial (Liu et al., 2012). Cyclophilin A enhances the interaction between RIG-I and TRIM25 during the infection of some viruses, such as Sendai virus. NDR2 directly interacts with RIG-I and TRIM25 and promotes the K63linked polyubiquitination of RIG-I mediated by TRIM25 (Liu et al., 2019). TRIM25 also interacts with NLRP12 resulting in disrupting Lys63 ubiquitination and activation of RIG-I (Chen et al., 2019). Other E3 ligases like TRIM4 and MEX3C (Mex3 RNA binding family member C) have also been shown to conjugate covalent K63-linked polyubiquitin chains to RIG-I and mediate its activation (Davis and Gack, 2015). Alternatively, RIGI also undergoes K48-linked ubiquitination by RNF125, RNF122 (ring finger protein 125) (Jia et al., 2017) and c-Cbl (Casitas B-lineage lymphoma proto-oncogene) (Chen et al., 2013) for proteasomal degradation to form a negative feedback loop of RIG-I signaling. Ubiquitination-dependent signaling was also reported in MDA5 activation. TRIM65 is responsible for K63linked ubiquitination at Lys743 of MDA5 and arrestin domain containing 4 (ARRDC4) interacts with MDA5 to recruit the E3 ligase (Lang et al., 2017).

Several deubiquitinating enzymes have been demonstrated to function in the RLRs activation process. If the K48-linked ubiquitin chains are eliminated, it will promote signaling activation, such as the function of ubiquitin specific peptidase 4 (USP4) and ovarian tumor domain-containing ubiquitin aldehyde binding protein 1 (OTUB1) (Wang et al., 2013a; Jahan et al., 2020). Conversely, multiple proteins have been identified to deubiquitinate K63-linked ubiquitin from RLRs, which results in signaling inhibition (Friedman et al., 2008; Cui et al., 2014; Fan et al., 2014; Lin et al., 2016; Tao et al., 2020).

Phosphorylation of RLRs (extensively investigated in RIG-I and MDA5) mainly shows inhibitory effects on their activation. Phosphorylation on CARDs (at S8 and T170) and the CTD (at T770 and S854/855) of RIG-I and on MAD5 (at S88 and S828) suppresses the activation of these sensors, if not all, most of which are removed during their activation (Gack et al., 2010; NistalVillan et al., 2010; Sun et al., 2011). Dynamic sumoylation and desumoylation of MDA5 and RIG-I have also been reported. In uninfected cells or at the early stage of infection (Sendai virus infection), the sumoylation of MDA5 and RIG-I suppresses their K48-linked polyubiquitination and degradation. At the late phase of the viral infection, the modification is removed by a host factor, SENP2 (sentrin/sumo-specific protease 2), which results in their K48-linked polyubiquitination and degradation of these RLRs (Hu et al., 2017). ISG15 (IFN-stimulated gene 15) is a small ubiquitin-like protein that could be covalently conjugated onto RLRs, and this modification (ISGylation) also contributes to activation of the sensors (Ketscher et al., 2015;
Villarroya-Beltri et al., 2017; Zhang et al., 2017; Perng and Lenschow, 2018). Just like ubiquitination, ISGylation is also a reversible process that could be removed by ubiquitinspecific peptidase 18 (USP18) protein (Malakhov et al., 2002; Ritchie et al., 2017).

\section{DNA VIRUS DETECTION BY RLRS}

Functions of the cytoplasmic DNA sensor, cyclic GMP-AMP synthase (cGAS) during the infection of several DNA viruses have been exclusively investigated (Hopfner and Hornung, 2020). Interestingly, in some cases of DNA virus infection, stimulation of innate immune reaction also depends on RLR/MAVS signaling activation (Chan and Gack, 2016). The most intriguing question about this phenomenon was how the immunostimulatory RNAs are generated during DNA virus infection. Now, we know these RNAs could be the transcripts either from cellular or foreign genomes.

In physiological conditions, cytoplasm DNA existence should be avoided. Nevertheless, under some circumstances, such as autoimmune responses or virus infection, host DNA and/or foreign DNA would be inappropriately delivered into the cytosol, and this DNA could be transcribed by cytoplasmically localized Type III RNA polymerase (RNAPIII) (Chiu et al., 2009). Though three main RNA polymerases are expressed in eukaryotic cells, only type III is abundant in the cytosol. RNAPIII is also the largest RNA polymerase currently known and consists of 17 subunits, including DNA binding sites, which can catalyze gene transcription (Hoffmann et al., 2015). Initially, RNAPIII was reported as a sensor for cytoplasmic DNA during DNA virus infection (Ablasser et al., 2009; Luke et al., 2011). It can also transcribe short-spread and repetitive elements in the human genome. During innate immune response, RNAPIII synthesizes 5S rRNA, tRNA, and other small RNAs through a specific promoter region, taking the dsDNA-rich AT as a template to transcribe into $5^{\prime}$-triphosphate dsRNA, which is the RIG-I ligand (Ablasser et al., 2009; Chiu et al., 2009; Luke et al., 2011). Likely, the viral genome containing the ATrich features could induce RNAPIII-dependent RNA synthesis (Ablasser et al., 2009; Chiu et al., 2009). Consistent with this, AT-rich DNA sequences could be transcribed during ionizing radiation treatment and initiate MAVS-dependent RNA sensing and signaling (Feng et al., 2020).

In addition to being present and functioning in the cytosol, RNAPIII is also a nucleus resident who transcribes RNAs to activate RLR/MAVS signaling, as illustrated during Epstein-Barr virus (EBV) infection (Rosa et al., 1981). After infection, the virus establishes life-long latency in the host cell, and only a small set of viral transcripts are produced. EBV-encoded small RNAs (EBERs) transcribed by RNAPIII, which consists of EBER1 and EBER2, are the most abundant viral transcripts during EBV latency. These EBERs contain biochemical features that could be recognized by RIG-I and are sufficient to activate RLR/MAVS signaling (Glickman et al., 1988; Samanta et al., 2006, 2008). Furthermore, the exosome-dependent transition of EBER1 was identified. EBER1 could be sensed by both TLR-3 and RIG-I 
in target cells, which is also dependent on the presence of its 5'-triphosphate moiety (Iwakiri et al., 2009; Baglio et al., 2016).

The importance of RNAPIII in controlling varicella-zoster virus (VZV) infection is revealed in a study showing that mutation in the RNAPIII machinery results in severe illness (Ogunjimi et al., 2017). Furthermore, inhibition of RNAPIII with ML-60218 reduced IFN- $\beta$ production during HSV-1, EBV, and adenovirus infection, but not an RNA virus, Sendai virus infection (Chiu et al., 2009). Virus-associated RNAs (VAs) were confirmed in Adenovirus infection for RIG-I downstream signaling activation. These small non-coding RNAs are highly similar to the EBV-encoded EBERs (Rosa et al., 1981). Whether the similar RNAs activate the signaling in HSV infection needs further investigation. The expression of type I interferon induced by adenovirus VAs also coincides with the VA-RNA expression phase during the virus infection (Minamitani et al., 2011). Furthermore, it seems that VA functions differently in different cell types. The production of type I IFN in MEFs was completely dependent on RLR/MAVS signaling, whereas, in generated bone marrow-derived dendritic cells (GM-DCs) it was partially dependent (Yamaguchi et al., 2010). Since adenovirus vector is used widely in fundamental research and clinical practice, these studies should advance the vector designs (Machitani et al., 2011; Darweesh et al., 2019).

Aside from the immunostimulatory RNAs transcribed in the cytoplasm, transcripts of retrotransposons and pseudogenes from the host genome that can activate RLR/MAVS have also been identified in DNA virus infection. During murine gammaherpesvirus 68 (MHV-68) infection, SINE (short interspersed nuclear element) RNAs are rapidly transcribed by RNAPIII in the nucleus and activate the antiviral NF- $\kappa \mathrm{B}$ signaling pathway through MAVS-dependent and independent mechanisms (John et al., 2015). HSV-1 infection induces relocalization of $5 \mathrm{~S}$ ribosomal RNA pseudogene 141 (RNA5SP141) from the nucleus to the cytoplasm that binds to RIG-I and activates the downstream signaling (Kang et al., 2004). HSV-1 infection could induce the shutoff of host protein synthesis, such as ribosomal protein L5 (RPL5). RPL5 associates with 5S rRNA to form 5S ribonucleoprotein particles. Both mitochondrial ribosomal protein L18 (MRPL18) and thiosulfate sulfurtransferase (TST) facilitate the import of cytoplasmic 5S rRNA into mitochondria (Zhang et al., 2007; Smirnov et al., 2010, 2011). HSV-1mediated shutoff of these protein synthesis could liberate and relocate RNA5SP141 in cytoplasm for recognition by RIG-I (Chiang J.J. et al., 2018).

Vault RNAs (vtRNAs) are another type of host small RNAs. They initially contain a $5^{\prime}$-triphosphate after being transcribed by RNAPIII, and the $5^{\prime}$-triphosphate moieties are removed by the cellular triphosphatase, dual-specificity phosphatase 11 (DUSP11), in uninfected cells resulting in the conversion of these RNAs to be non-immunostimulatory (Zhao et al., 2018). During KSHV infection, the expression of this type of RNAs are enhanced. Furthermore, KSHV infection reduces the expression of host DUSP11, which leads to the accumulation of immunostimulatory RNAs (Nottingham et al., 2016; Yang et al., 2018). A recent study also showed that transcripts from multiple
KSHV genomic regions bind to and activate RIG-I during the virus infection (Zhang et al., 2018).

\section{EVASION OF THE RLR SIGNALING PATHWAY BY DNA VIRUSES}

Although RLR-mediated innate immune response could elicit a robust immune response against incoming DNA viruses, these viruses have adopted multiple evasion mechanisms for counteracting and escaping such immune responses. RLR recognition of PAMPs, responsible for initiating antiviral signaling, is a target for these viruses. Damping of RLRs and host factors participating in RLR activation regulation in the signaling pathway is one of the key mechanisms for these viruses' evasion. For example, EBV can promote RIG-I degradation through the proteasomal pathway by recruiting an E3 ubiquitin ligase, the carboxyl-terminus of Hsp70 interacting protein (CHIP) to the RIG-I through EBV-encoded LMP1 (Chongfeng et al., 2018). Our previous studies found that human herpesvirus 6 (HHV6), a DNA virus, can regulate the RLR/MAVS signaling pathway by affecting the mitochondrial membrane potential (Jiang et al., 2021). Here, we summarize the strategies that DNA viruses employed to evade the RLRs-mediated innate antiviral responses. Eliminating the sensor or a factor in the signaling pathway is one of the effective mechanisms to suppress the immune responses. Many viruses encode viral proteases that directly cleave RLR, as mentioned above of EBV infection (Baglio et al., 2016). Since MAVS is critical for both RIG-I- and MDA5mediated signaling, many viral products, such as HCMV US9 and HHV-6 U26 proteins, target and cleave MAVS by inducing the leakage of mitochondrial MAVS for degradation (Choi et al., 2018; Jiang et al., 2021). Moreover, during HCMV infection RIG-I was significantly degraded (Scott, 2009). Hepatitis B virus (HBV) also produces RNA species during its life cycle, which binds to MAVS and promotes its degradation through Lys136 ubiquitination (Sato et al., 2015). A human herpesvirus 8 (HHV-8) gene product, viral interferon regulatory factor 1 (vIRF-1) is targeted to the mitochondrial detergent-resistant membrane (mDRM) during virus replication. It was shown that vIRF-1 negatively regulates the MAVS-mediated antiviral responses (Lin et al., 2001; Hwang and Choi, 2016). And the interaction of HSV-1 US11 protein with RIG-I and MAVS results in the impediment of RIG-I/MAVS and MDA5/MAVS complex formation and subsequent reduced production of IFN- $\beta$ (Xing et al., 2012; Su et al., 2016; Zhu and Zheng, 2020). Other strategies may also be taken by HSV-1 for the evasion of RLR detection (Wang et al., 2013b) and the subsequent signaling (Zhu et al., 2011; Wang et al., 2013c, 2014; Xing et al., 2013; Zhang et al., 2013).

Manipulation of post-translational modification of RLRs is another key strategy for immune evasion. As described above, reversed ubiquitination is an important step in regulating RLR signaling cascade. Many DNA viruses attenuate IFN response by altering the ubiquitination of RLRs and/or RLR-regulatory proteins through various mechanisms. Since the E3 ligase TRIM25 is a key activator of the RIG-I-mediated signaling 


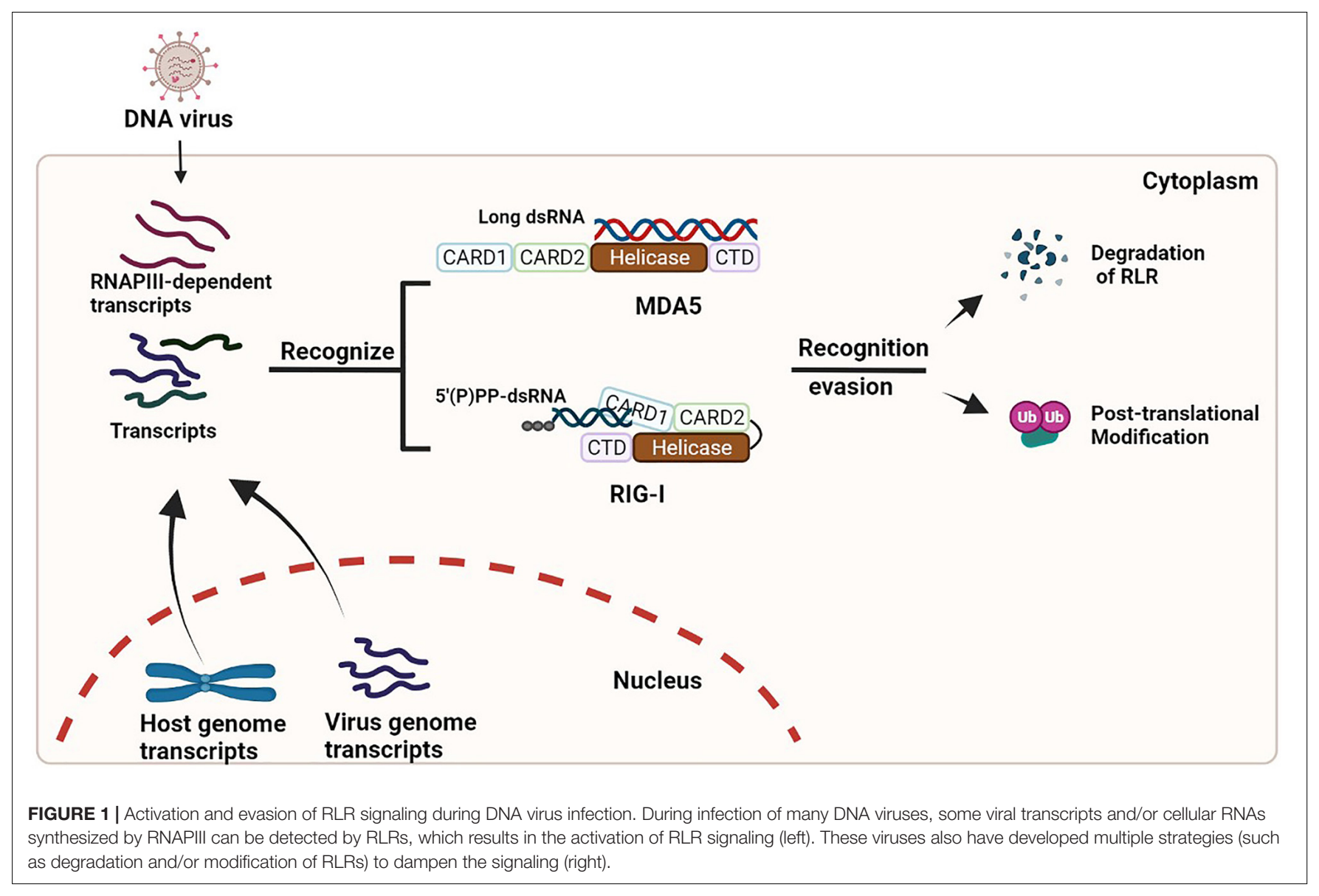

pathway (Castanier et al., 2012), many viruses, including DNA viruses, target this protein for immune evasion. During human papillomavirus (HPV) infection, the E6 oncoprotein interacts with TRIM25 and ubiquitin-specific peptidase 15 (USP15), which enhances TRIM25 degradation and subsequently inhibits RLR/MAVS signaling. These interactions promote the ubiquitination and degradation of TRIM25. Moreover, these processes inhibit the ubiquitination associated with RIG-I and K63, block RIG-I-MAVS interactions, and ultimately prevent the activation of RIG-I-mediated signaling (Chiang C. et al., 2018). KSHV encodes a viral deubiquitinating enzyme (DUB), ORF64. This viral enzyme effectively removes both K48-linked and K63linked ubiquitin chains from RIG-I, which inhibits the activation of RLR/MAVS signaling (González et al., 2009; Inn et al., 2011). DNA viruses contain a relatively large genome compared with RNA viruses. There should be no doubt that other strategies, such as manipulating cellular DUB expression, used by many RNA viruses, would also be used by DNA viruses.

\section{CONCLUDING REMARKS AND FUTURE PERSPECTIVES}

In recent years, the roles of activating the RLR/MAVS signaling pathway during DNA virus infection has been identified.
However, the genome features of these DNA viruses are not readily for RLR recognition. Some of the viral transcripts and the unusual cellular RNAs (mislocated or unmarked) can be recognized and bound by RLRs, which could subsequently trigger an antiviral innate immune response that is proven to play an important role in controlling viral infections. Meanwhile, almost all these viruses have also developed individual strategies to escape RLR/MAVS-mediated antiviral response, which needs to be elucidated in the contexts of each virus infection (summarized in Figure 1).

A large amount of studies in this field has been focused on elucidating mechanisms of immunostimulatory RNA ligands for RLR recognition, modification of key components of the RLR/MAVS pathway, and the evasion strategies usurped by the viruses. Additional studies for most DNA viruses have to be conducted to investigate whether RLR/MAVS signaling activation is a general phenomenon and the physiological roles of the signaling in the disease contexts. Furthermore, it is worth to clarifying whether and how RLR/MAVS signaling cooperates with other innate immune signaling.

\section{AUTHOR CONTRIBUTIONS}

All authors listed have made a substantial, direct, and intellectual contribution to the work, and approved it for publication. 


\section{FUNDING}

This work was supported by the National Natural Science Foundation of China (grants 81571979 and 82172263).

\section{REFERENCES}

Ablasser, A., and Sun, H. (2019). Regulation of cGAS- and RLR-mediated immunity to nucleic acids. Nat. Immunol. 21, 17-29. doi: 10.1038/s41590-019-0556-1

Ablasser, A., Bauernfeind, F., Hartmann, G., Latz, E., Fitzgerald, K. A., and Hornung, V. (2009). RIG-I-dependent sensing of poly(dA:dT) through the induction of an RNA polymerase III-transcribed RNA intermediate. Nat. Immunol. 10, 1065-U1040. doi: 10.1038/ni.1779

Andrejeva, J., Childs, K. S., Young, D. F., Carlos, T. S., Stock, N., Goodbourn, S., et al. (2004). The V proteins of paramyxoviruses bind the IFN-inducible RNA helicase, mda-5, and inhibit its activation of the IFN-beta promoter. Proc. Natl. Acad. Sci. U.S.A. 101, 17264-17269. doi: 10.1073/pnas.0407639101

Baglio, S. R., van Eijndhoven, M. A. J., Koppers-Lalic, D., Berenguer, J., Lougheed, S. M., Gibbs, S., et al. (2016). Sensing of latent EBV infection through exosomal transfer of 5'pppRNA. Proc. Natl. Acad. Sci. U.S.A. 113, 587-596. doi: 10.1073/ pnas. 1518130113

Ban, J., Lee, N.-R., Lee, N.-J., Lee, J. K., Quan, F.-S., and Inn, K.-S. (2018). Human respiratory syncytial virus NS 1 targets TRIM25 to Suppress RIGI ubiquitination and subsequent RIG-I-mediated antiviral signaling. Viruses 10:716. doi: $10.3390 / \mathrm{v} 10120716$

Berke, I. C., and Modis, Y. (2012). MDA5 cooperatively forms dimers and ATPsensitive filaments upon binding double-stranded RNA. Embo J. 31, 1714-1726. doi: 10.1038/emboj.2012.19

Bruns, A. M., Leser, G. P., Lamb, R. A., and Horvath, C. M. (2014). The innate immune sensor LGP2 activates antiviral signaling by regulating MDA5-RNA interaction and filament assembly. Mol. Cell 55, 771-781. doi: 10.1016/j.molcel. 2014.07.003

Cardenas, W. B., Loo, Y.-M., Gale, M. Jr., Hartman, A. L., Kimberlin, C. R., Martinez-Sobrido, L., et al. (2006). Ebola virus VP35 protein binds doublestranded RNA and inhibits alpha/beta interferon production induced by RIG-I signaling. J. Virol. 80, 5168-5178. doi: 10.1128/jvi.02199-05

Castanier, C., Zemirli, N., Portier, A., Garcin, D., Bidere, N., Vazquez, A., et al. (2012). MAVS ubiquitination by the E3 ligase TRIM25 and degradation by the proteasome is involved in type I interferon production after activation of the antiviral RIG-I-like receptors. Bmc Biol. 10:44. doi: 10.1186/1741-7007-10-44

Chan, Y. K., and Gack, M. U. (2016). Viral evasion of intracellular DNA and RNA sensing. Nat. Rev. Microbiol. 14, 360-373. doi: 10.1038/nrmicro.2016.45

Chen, S.-T., Chen, L., Lin, D. S.-C., Chen, S.-Y., Tsao, Y.-P., Guo, H., et al. (2019). NLRP12 regulates anti-viral RIG-I activation via interaction with TRIM25. Cell Host Microbe 25, 602-616. doi: 10.1016/j.chom.2019.02.013

Chen, W., Han, C., Xie, B., Hu, X., Yu, Q., Shi, L., et al. (2013). Induction of siglec$\mathrm{G}$ by RNA viruses inhibits the innate immune response by promoting RIG-I degradation. Cell 152, 467-478. doi: 10.1016/j.cell.2013.01.011

Chiang, C., Pauli, E.-K., Biryukov, J., Feister, K. F., Meng, M., White, E. A., et al. (2018). The human papillomavirus E6 oncoprotein targets USP15 and TRIM25 To suppress RIG-I-mediated innate immune signaling. J. Virol. 92, e01737-e01817. doi: 10.1128/jvi.01737-17

Chiang, J. J., Sparrer, K. M. J., van Gent, M., Laessig, C., Huang, T., Osterrieder, N., et al. (2018). Viral unmasking of cellular 5S rRNA pseudogene transcripts induces RIG-I-mediated immunity. Nat. Immunol. 19, 53-62. doi: 10.1038/ s41590-017-0005-y

Chiu, Y.-H., MacMillan, J. B., and Chen, Z. J. (2009). RNA polymerase III detects cytosolic DNA and induces type I interferons through the RIG-I pathway. Cell 138, 576-591. doi: 10.1016/j.cell.2009.06.015

Choi, H. J., Park, A., Kang, S., Lee, E., Lee, T. A., Ra, E. A., et al. (2018). Human cytomegalovirus-encoded US9 targets MAVS and STING signaling to evade type I interferon immune responses. Nat. Commun. 9:125. doi: 10.1038/s41467017-02624-8

Chongfeng, X., Lei, S., Wenjun, L., and Ziyuan, D. (2018). Latent membrane protein 1 of epstein-barr virus promotes RIG-I degradation mediated by proteasome pathway. Front. Immunol. 9:1446. doi: 10.3389/fimmu.2018.01446

\section{ACKNOWLEDGMENTS}

We would like to thank Garbarino Emanuela for editing this manuscript.

Cui, J., Song, Y., Li, Y., Zhu, Q., Tan, P., Qin, Y., et al. (2014). USP3 inhibits type I interferon signaling by deubiquitinating RIG-I-like receptors. Cell Res. 24, 400-416. doi: 10.1038/cr.2013.170

Darweesh, M., Kamel, W., Gavrilin, M. A., Akusjarvi, G., and Svensson, C. (2019). Adenovirus VA RNAI blocks ASC oligomerization and inhibits NLRP3 inflammasome activation. Front. Immunol. 10:2791. doi: 10.3389/fimmu.2019. 02791

Davis, M. E., and Gack, M. U. (2015). Ubiquitination in the antiviral immune response. Virology 47, 52-65. doi: 10.1016/j.virol.2015.02.033

Decroly, E., Ferron, F., Lescar, J., and Canard, B. (2012). Conventional and unconventional mechanisms for capping viral mRNA. Nat. Rev. Microbiol. 10, 51-65. doi: 10.1038/nrmicro2675

Devarkar, S. C., Schweibenz, B., Wang, C., Marcotrigiano, J., and Patel, S. S. (2018). RIG-I uses an ATPase-powered translocation-throttling mechanism for kinetic proofreading of RNAs and oligomerization. Mol. Cell 72, 355-368. doi: 10.1016/ j.molcel.2018.08.021

Du, Y., Duan, T., Feng, Y., Liu, Q., Lin, M., Cui, J., et al. (2018). LRRC25 inhibits type I IFN signaling by targeting ISG15-associated RIG-I for autophagic degradation. Embo J. 37, 351-366. doi: 10.15252/embj.201796781

Duic, I., Tadakuma, H., Harada, Y., Yamaue, R., Deguchi, K., Suzuki, Y., et al. (2020). Viral RNA recognition by LGP2 and MDA5, and activation of signaling through step-by-step conformational changes. Nucleic Acids Res. 48, 1166411674. doi: 10.1093/nar/gkaa935

Fan, Y., Mao, R., Yu, Y., Liu, S., Shi, Z., Cheng, J., et al. (2014). USP21 negatively regulates antiviral response by acting as a RIG-I deubiquitinase. J. Exp. Med. 211, 313-328. doi: 10.1084/jem.20122844

Feng, X., Tubbs, A., Zhang, C. C., Tang, M. F., Sridharan, S., Wang, C., et al. (2020). ATR inhibition potentiates ionizing radiation-induced interferon response via cytosolic nucleic acid-sensing pathways. Embo J. 39:e104036. doi: 10.15252/ embj.2019104036

Friedman, C. S., O’Donnell, M. A., Legarda-Addison, D., Ng, A., Cárdenas, W. B., Yount, J. S., et al. (2008). The tumour suppressor CYLD is a negative regulator of RIG-I-mediated antiviral response. EMBO Rep. 9, 930-936. doi: 10.1038/ embor.2008.136

Gack, M. U., Albrecht, R. A., Urano, T., Inn, K.-S., Huang, I. C., Carnero, E., et al. (2009). Influenza A virus NS1 targets the ubiquitin ligase TRIM25 to evade recognition by the host viral RNA sensor RIG-I. Cell Host Microbe 5, 439-449. doi: 10.1016/j.chom.2009.04.006

Gack, M. U., Nistal-Villan, E., Inn, K. S., Garcia-Sastre, A., and Jung, J. U. (2010). Phosphorylation-mediated negative regulation of RIG-I antiviral activity. J. Virol. 84, 3220-3229. doi: 10.1128/jvi.02241-09

Gack, M. U., Shin, Y. C., Joo, C.-H., Urano, T., Liang, C., Sun, L., et al. (2007). TRIM25 RING-finger E3 ubiquitin ligase is essential for RIG-I-mediated antiviral activity. Nature 446, 916-920. doi: 10.1038/nature05732

Glickman, J. N., Howe, J. G., and Steitz, J. A. (1988). Structural analyses of EBER1 and EBER2 ribonucleoprotein particles present in epstein-barr virus-infected cells. J. Virol. 62, 902-911. doi: 10.1128/jvi.62.3.902-911.1988

González, C. M., Wang, L., and Damania, B. (2009). Kaposi's sarcoma-associated herpesvirus encodes a viral deubiquitinase. J. Virol. 83, 10224-10233. doi: 10. 1128/jvi.00589-09

Goubau, D., Deddouche, S., and Reis e Sousa, C. (2013). Cytosolic sensing of viruses. Immunity 38, 855-869. doi: 10.1016/j.immuni.2013.05.007

Hoffmann, N. A., Jakobi, A. J., Moreno-Morcillo, M., Glatt, S., Kosinski, J., Hagen, W. J. H., et al. (2015). Molecular structures of unbound and transcribing RNA polymerase III. Nature 528, 231-236. doi: 10.1038/nature16143

Hopfner, K. P., and Hornung, V. (2020). Molecular mechanisms and cellular functions of cGAS-STING signalling. Nat. Rev. Mol. Cell Biol. 21, 501-521. doi: 10.1038/s41580-020-0244-x

Hornung, V., Ellegast, J., Kim, S., Brzozka, K., Jung, A., Kato, H., et al. (2006). 5 '-triphosphate RNA is the ligand for RIG-I. Science 314, 994-997. doi: 10.1126/ science. 1132505 
Hu, M. M., Liao, C. Y., Yang, Q., Xie, X. Q., and Shu, H. B. (2017). Innate immunity to RNA virus is regulated by temporal and reversible sumoylation of RIG-I and MDA5. J. Exp. Med. 214, 973-989. doi: 10.1084/jem.20161015

Hwang, K. Y., and Choi, Y. B. (2016). Modulation of mitochondrial antiviral signaling by human herpesvirus 8 interferon regulatory factor 1. J. Virol. 90 , 506-520. doi: 10.1128 /jvi.01903-15

Inn, K. S., Lee, S. H., Rathbun, J. Y., Wong, L. Y., Toth, Z., Machida, K., et al. (2011). Inhibition of RIG-I-mediated signaling by Kaposi's sarcoma-associated herpesvirus-encoded deubiquitinase ORF64. J. Virol. 85, 10899-10904. doi: 10. 1128/jvi.00690-11

Iwakiri, D., Zhou, L., Samanta, M., Matsumoto, M., Ebihara, T., Seya, T., et al. (2009). Epstein-Barr virus (EBV)-encoded small RNA is released from EBVinfected cells and activates signaling from toll-like receptor 3. J. Exp. Med. 206, 2091-2099. doi: 10.1084/jem.20081761

Jahan, A. S., Biquand, E., Muñoz-Moreno, R., Le Quang, A., Mok, C. K., Wong, H. H., et al. (2020). OTUB1 Is a key regulator of RIG-I-dependent immune signaling and is targeted for proteasomal degradation by influenza A NS1. Cell Rep. 30, 1570.e-1584.e. doi: 10.1016/j.celrep.2020.01.015

Jia, X., Zhou, H., Wu, C., Wu, Q., Ma, S., Wei, C., et al. (2017). The ubiquitin ligase RNF125 targets innate immune adaptor protein TRIM14 for ubiquitination and degradation. J. Immunol. 198, 4652-4658. doi: 10.4049/jimmunol.1601322

Jiang, X., Tang, T., Guo, J., Wang, Y., and Tang, H. (2021). Human herpesvirus $6 \mathrm{~B}$ U26 inhibits the activation of the RLR/MAVS signaling pathway. mBio 12, e03505-e03520. doi: 10.1128/mBio.03505-20

John, K., Emma, A., Glaunsinger, B. A., and Robertson, E. S. (2015). Infectioninduced retrotransposon-derived noncoding RNAs enhance herpesviral gene expression via the NF-KB pathway. PLoS Pathog. 11:e1005260. doi: 10.1371/ journal.ppat.1005260

Kang, D. C., Gopalkrishnan, R. V., Lin, L., Randolph, A., Valerie, K., Pestka, S., et al. (2004). Expression analysis and genomic characterization of human melanoma differentiation associated gene-5, mda-5: a novel type I interferon-responsive apoptosis-inducing gene. Oncogene 23, 1789-1800. doi: 10.1038/sj.onc. 1207300

Kang, D. C., Gopalkrishnan, R. V., Wu, Q., Jankowsky, E., Pyle, A. M., and Fisher, P. B. (2002). Mda-5: an interferon-inducible putative RNA helicase with double-stranded RNA-dependent ATPase activity and melanoma growthsuppressive properties. Proc. Natl. Acad. Sci. U.S.A. 99, 637-642. doi: 10.1073/ pnas.022637199

Kato, H., Takeuchi, O., Mikamo-Satoh, E., Hirai, R., Kawai, T., Matsushita, K., et al. (2008). Length-dependent recognition of double-stranded ribonucleic acids by retinoic acid-inducible gene-I and melanoma differentiation-associated gene 5 . J. Exp. Med. 205, 1601-1610. doi: 10.1084/jem.20080091

Kato, H., Takeuchi, O., Sato, S., Yoneyama, M., Yamamoto, M., Matsui, K., et al. (2006). Differential roles of MDA5 and RIG-I helicases in the recognition of RNA viruses. Nature 441, 101-105. doi: 10.1038/nature 04734

Kawai, T., Takahashi, K., Sato, S., Coban, C., Kumar, H., Kato, H., et al. (2005). IPS1 , an adaptor triggering RIG-I- and Mda5-mediated type I interferon induction. Nat. Immunol. 6, 981-988. doi: 10.1038/ni1243

Ketscher, L., Hannss, R., Morales, D. J., Basters, A., Guerra, S., Goldmann, T., et al. (2015). Selective inactivation of USP18 isopeptidase activity in vivo enhances ISG15 conjugation and viral resistance. Proc. Natl. Acad. Sci. U.S.A. 112, 1577-1582. doi: 10.1073/pnas.1412881112

Koliopoulos, M. G., Lethier, M., van der Veen, A. G., Haubrich, K., Hennig, J., Kowalinski, E., et al. (2018). Molecular mechanism of influenza A NS1mediated TRIM25 recognition and inhibition. Nat. Commun. 9:1820. doi: 10. 1038/s41467-018-04214-8

Komuro, A., and Horvath, C. M. (2006). RNA- and virus-independent inhibition of antiviral signaling by RNA helicase LGP2. J. Virol. 80, 12332-12342. doi: 10.1128/jvi.01325-06

Kowalinski, E., Lunardi, T., McCarthy, A. A., Louber, J., Brunel, J., Grigorov, B., et al. (2011). Structural basis for the activation of innate immune patternrecognition receptor RIG-I by viral RNA. Cell 147, 423-435. doi: 10.1016/j.cell. 2011.09.039

Lang, X., Tang, T., Jin, T., Ding, C., Zhou, R., and Jiang, W. (2017). TRIM65catalized ubiquitination is essential for MDA5-mediated antiviral innate immunity. J. Exp. Med. 214, 459-473. doi: 10.1084/jem.20160592
Li, X., Ranjith-Kumar, C. T., Brooks, M. T., Dharmaiah, S., Herr, A. B., Kao, C., et al. (2009). The RIG-I-like receptor LGP2 recognizes the termini of doublestranded RNA. J. Biol. Chem. 284, 13881-13891. doi: 10.1074/jbc.M900818200

Lin, R., Genin, P., Mamane, Y., Sgarbanti, M., Battistini, A., Harrington, W. J., et al. (2001). HHV-8 encoded vIRF-1 represses the interferon antiviral response by blocking IRF-3 recruitment of the CBP/p300 coactivators. Oncogene 20 , 800-811. doi: 10.1038/sj.onc. 1204163

Lin, W., Zhang, J., Lin, H., Li, Z., Sun, X., Xin, D., et al. (2016). Syndecan-4 negatively regulates antiviral signalling by mediating RIG-I deubiquitination via CYLD. Nat. Commun. 7:11848. doi: 10.1038/ncomms11848

Liu, G., and Gack, M. U. (2020). Distinct and orchestrated functions of RNA sensors in innate immunity. Immunity 53, 26-42. doi: 10.1016/j.immuni.2020. 03.017

Liu, H. M., Loo, Y.-M., Horner, S. M., Zornetzer, G. A., Katze, M. G., and Gale, M. Jr. (2012). The mitochondrial targeting chaperone 14-3-3 epsilon regulates a RIG-I translocon that mediates membrane association and innate antiviral immunity. Cell Host Microbe 11, 528-537. doi: 10.1016/j.chom.2012.04.006

Liu, T. X., Zhang, J. W., Tao, J., Zhang, R. B., and Chen, Z. (2000). Gene expression networks underlying retinoic acid-induced differentiation of acute promyelocytic leukemia cells. Blood 96, 1496-1504. doi: 10.1182/blood.V96.4. 1496

Liu, Z., Wu, C., Pan, Y., Liu, H., Wang, X., Yang, Y., et al. (2019). NDR2 promotes the antiviral immune response via facilitating TRIM25-mediated RIG-I activation in macrophages. Sci. Adv. 5:eaav0163. doi: 10.1126/sciadv. aav0163

Luke, J. M., Simon, G. G., Soederholm, J., Errett, J. S., August, T., Gale, M. Jr., et al. (2011). Coexpressed RIG-I agonist enhances humoral immune response to influenza virus DNA vaccine. J. Virol. 85, 1370-1383. doi: 10.1128/jvi.01250-10

Luo, D., Ding, S. C., Vela, A., Kohlway, A., Lindenbach, B. D., and Pyle, A. M. (2011). Structural insights into RNA recognition by RIG-I. Cell 147, 409-422. doi: 10.1016/j.cell.2011.09.023

Luo, D., Kohlway, A., and Pyle, A. M. (2013). Duplex RNA activated ATPases (DRAs): platforms for RNA sensing, signaling and processing. RNA Biol. 10, 111-120. doi: 10.4161/rna.22706

Machitani, M., Yamaguchi, T., Shimizu, K., Sakurai, F., Katayama, K., Kawabata, K., et al. (2011). Adenovirus vector-derived VA-RNA-mediated innate immune responses. Pharmaceutics 3, 338-353. doi: 10.3390/pharmaceutics3030338

Malakhov, M. P., Malakhova, O. A., Kim, K. I., Ritchie, K. J., and Zhang, D. E. (2002). UBP43 (USP18) specifically removes ISG15 from conjugated proteins. J. Biol. Chem. 277, 9976-9981. doi: 10.1074/jbc.M109078200

Meylan, E., Curran, J., Hofmann, K., Moradpour, D., Binder, M., Bartenschlager, R., et al. (2005). Cardif is an adaptor protein in the RIG-I antiviral pathway and is targeted by hepatitis C virus. Nature 437, 1167-1172. doi: 10.1038/nature04193

Minamitani, T., Iwakiri, D., and Takada, K. (2011). Adenovirus virus-associated RNAs induce type I interferon expression through a RIG-I-Mediated pathway. J. Virol. 85, 4035-4040. doi: 10.1128/jvi.02160-10

Myong, S., Sheng, C., Cornish, P. V., Kirchhofer, A., Gack, M. U., Jung, J. U., et al. (2009). Cytosolic viral sensor RIG-I Is a 5'-triphosphate-dependent translocase on double-stranded RNA. Science 323, 1070-1074. doi: 10.1126/ science. 1168352

Nistal-Villan, E., Gack, M. U., Martinez-Delgado, G., Maharaj, N. P., Inn, K. S., Yang, H., et al. (2010). Negative role of RIG-I serine 8 phosphorylation in the regulation of interferon-beta production. J. Biol. Chem. 285, 20252-20261. doi: $10.1074 /$ jbc.M109.089912

Nottingham, R. M., Burke, J. M., Kincaid, R. P., Lambowitz, A. M., and Sullivan, C. S. (2016). DUSP11 activity on triphosphorylated transcripts promotes argonaute association with noncanonical viral microRNAs and regulates steady-state levels of cellular noncoding RNAs. Genes Dev. 30, 2076-2092. doi: $10.1101 /$ gad.282616.116

Ogunjimi, B., Zhang, S. Y., Sorensen, K. B., Skipper, K. A., Carter-Timofte, M., Kerner, G., et al. (2017). Inborn errors in RNA polymerase III underlie severe varicella zoster virus infections. J. Clin. Invest. 127, 3543-3556. doi: 10.1172/ JCI92280

Onomoto, K., Onoguchi, K., and Yoneyama, M. (2021). Regulation of RIG-I-like receptor-mediated signaling: interaction between host and viral factors. Cell. Mol. Immunol. 18, 539-555. doi: 10.1038/s41423-020-00602-7 
Peisley, A., Jo, M. H., Lin, C., Wu, B., Orme-Johnson, M., Walz, T., et al. (2012). Kinetic mechanism for viral dsRNA length discrimination by MDA5 filaments. Proc. Natl. Acad. Sci. U.S.A. 109, 3340-3349. doi: 10.1073/pnas.1208618109

Peisley, A., Wu, B., Xu, H., Chen, Z. J., and Hur, S. (2014). Structural basis for ubiquitin-mediated antiviral signal activation by RIG-I. Nature 509, 110-114. doi: 10.1038/nature13140

Peisley, A., Wu, B., Yao, H., Walz, T., and Hur, S. (2013). RIG-I forms signalingcompetent filaments in an ATP-Dependent, Ubiquitin-Independent Manner. Mol. Cell 51, 573-583. doi: 10.1016/j.molcel.2013.07.024

Perng, Y. C., and Lenschow, D. J. (2018). ISG15 in antiviral immunity and beyond. Nat. Rev. Microbiol. 16, 423-439. doi: 10.1038/s41579-018-0020-5

Pichlmair, A., Schulz, O., Tan, C. P., Naslund, T. I., Liljestrom, P., Weber, F., et al. (2006). RIG-I-mediated antiviral responses to single-stranded RNA bearing 5 '-phosphates. Science 314, 997-1001. doi: 10.1126/science.1132998

Rehwinkel, J., and Gack, M. U. (2020). RIG-I-like receptors: their regulation and roles in RNA sensing. Nat. Rev. Immunol. 20, 537-551. doi: 10.1038/s41577020-0288-3

Ren, X., Linehan, M. M., Iwasaki, A., and Pyle, A. M. (2019). RIG-I selectively discriminates against 5 '-monophosphate RNA. Cell Rep. 26, 2019-2027. doi: 10.1016/j.celrep.2019.01.107

Ritchie, K. J., Chang, S. H., Kim, K. I., Ming, Y., and Zhang, D. E. (2017). Role of ISG15 protease UBP43 (USP18) in innate immunity to viral infection. Nat. Med. 10, 1374-1378. doi: 10.1038/nm1133

Rodriguez, K. R., and Horvath, C. M. (2014). Paramyxovirus V protein interaction with the antiviral sensor LGP2 disrupts MDA5 signaling enhancement but is not relevant to LGP2-mediated RLR signaling inhibition. J. Virol. 88, 81808188. doi: 10.1128/jvi.00737-14

Rosa, M. D., Gottlieb, E., Lerner, M. R., and Steitz, J. A. (1981). Striking similarities are exhibited by 2 small epstein-barr virus-encoded ribonucleic-acids and the adenovirus-associated ribonucleic-acids VAI and VAII. Mol. Cell. Biol. 1, 785-796. doi: 10.1128/mcb.1.9.785

Rothenfusser, S., Goutagny, N., DiPerna, G., Gong, M., Monks, B. G., Schoenemeyer, A., et al. (2005). The RNA helicase Lgp2 inhibits TLRindependent sensing of viral replication by retinoic acid-inducible gene-I. J. Immunol. 175, 5260-5268. doi: 10.4049/jimmunol.175.8.5260

Saito, T., Hirai, R., Loo, Y. M., Owen, D., Johnson, C. L., Sinha, S. C., et al. (2007). Regulation of innate antiviral defenses through a shared repressor domain in RIG-I and LGP2. Proc. Natl. Acad. Sci. U.S.A. 104, 582-587. doi: 10.1073/pnas. 0606699104

Samanta, M., Iwakiri, D., and Takada, K. (2008). Epstein-barr virus-encoded small RNA induces IL-10 through RIG-I-mediated IRF-3 signaling. Oncogene 27, 4150-4160. doi: 10.1038/onc.2008.75

Samanta, M., Iwakiri, D., Kanda, T., Imaizumi, T., and Takada, K. (2006). EB virusencoded RNAs are recognized by RIG-I and activate signaling to induce type IIFN. Embo J. 25, 4207-4214. doi: 10.1038/sj.emboj.7601314

Sato, S., Li, K., Kameyama, T., Hayashi, T., Ishida, Y., Murakami, S., et al. (2015). The RNA sensor RIG-I dually functions as an innate sensor and direct antiviral factor for Hepatitis B virus. Immunity 42, 123-132. doi: 10.1016/j.immuni.2014. 12.016

Satoh, T., Kato, H., Kumagai, Y., Yoneyama, M., Sato, S., Matsushita, K., et al. (2010). LGP2 is a positive regulator of RIG-I- and MDA5-mediated antiviral responses. Proc. Natl. Acad. Sci. U.S.A. 107, 1512-1517. doi: 10.1073/pnas. 0912986107

Schlee, M., Roth, A., Hornung, V., Hagmann, C. A., Wimmenauer, V., Barchet, W., et al. (2009). Recognition of 5 ' triphosphate by RIG-I helicase requires short blunt double-stranded RNA as contained in panhandle of negative-strand virus. Immunity 31, 25-34. doi: 10.1016/j.immuni.2009.05.008

Schmidt, A., Schwerd, T., Hamm, W., Hellmuth, J. C., Cui, S., Wenzel, M., et al. (2009). 5 '-triphosphate RNA requires base-paired structures to activate antiviral signaling via RIG-I. Proc.Natl. Acad. Sci. U.S.A. 106, 12067-12072. doi: 10.1073/pnas.0900971106

Schuberth-Wagner, C., Ludwig, J., Bruder, A. K., Herzner, A.-M., Zillinger, T., Goldeck, M., et al. (2015). A conserved histidine in the RNA sensor RIG-I controls immune tolerance to N-1-2 ' O-methylated Self RNA. Immunity 43, 41-51. doi: 10.1016/j.immuni.2015.06.015

Scott, I. (2009). Degradation of RIG-I following cytomegalovirus infection is independent of apoptosis. Microbes Infect. 11, 973-979. doi: 10.1016/j.micinf. 2009.07.001
Seth, R. B., Sun, L. J., Ea, C. K., and Chen, Z. J. J. (2005). Identification and characterization of MAVS, a mitochondrial antiviral signaling protein that activates NF-kappa B and IRF3. Cell 122, 669-682. doi: 10.1016/j.cell.2005.08. 012

Smirnov, A., Comte, C., Mager-Heckel, A. M., Addis, V., Krasheninnikov, I. A., Martin, R. P., et al. (2010). Mitochondrial enzyme rhodanese is essential for 5 S ribosomal RNA import into human mitochondria. J. Biol. Chem. 285, 30792-30803. doi: 10.1074/jbc.M110.151183

Smirnov, A., Entelis, N., Martin, R. P., and Tarassov, I. (2011). Biological significance of $5 \mathrm{~S}$ rRNA import into human mitochondria: role of ribosomal protein MRP-L18. Genes Dev. 25, 1289-1305. doi: 10.1101/gad.624711

Su, C., Zhan, G., and Zheng, C. (2016). Evasion of host antiviral innate immunity by HSV-1, an update. Virol. J. 13:38. doi: 10.1186/s12985-016-0495-5

Sun, Z. G., Ren, H. W., Liu, Y., Teeling, J. L., and Gu, J. (2011). Phosphorylation of RIG-I by casein kinase II inhibits its antiviral response. J. Virol. 85, 1036-1047. doi: 10.1128/jvi.01734-10

Tao, X., Chu, B., Xin, D., Li, L., and Sun, Q. (2020). USP27X negatively regulates antiviral signaling by deubiquitinating RIG-I. PLoS Pathog. 16:e1008293. doi: 10.1371/journal.ppat.1008293

Uchikawa, E., Lethier, M., Malet, H., Brunel, J., Gerlier, D., and Cusack, S. (2016). Structural analysis of dsRNA binding to anti-viral pattern recognition receptors LGP2 and MDA5. Mol. Cell 62, 586-602. doi: 10.1016/j.molcel.2016.04.021

Villarroya-Beltri, C., Guerra, S., and Sánchez-Madrid, F. (2017). ISGylation - a key to lock the cell gates for preventing the spread of threats. J. Cell Sci. 130, 2961-2969. doi: 10.1242/jcs.205468

Wang, K., Ni, L., Wang, S., and Zheng, C. (2014). Herpes simplex virus 1 protein kinase US3 hyperphosphorylates p65/RelA and dampens NF-kappa B activation. J. Virol. 88, 7941-7951. doi: 10.1128/jvi.03394-13

Wang, L., Zhao, W., Zhang, M., Wang, P., Zhao, K., Zhao, X., et al. (2013a). USP4 positively regulates RIG-I-mediated antiviral response through deubiquitination and stabilization of RIG-I. J. Virol. 87, 4507-4515. doi: 10. 1128/jvi.00031-13

Wang, S., Wang, K., Li, J., and Zheng, C. (2013b). Herpes simplex virus 1 ubiquitin-specific protease UL36 inhibits beta interferon production by deubiquitinating TRAF3. J. Virol. 87, 11851-11860. doi: 10.1128/jvi.01 211-13

Wang, S., Wang, K., Lin, R., and Zheng, C. (2013c). Herpes simplex virus 1 serine/threonine kinase US3 hyperphosphorylates IRF3 and inhibits beta interferon production. J. Virol. 87, 12814-12827. doi: 10.1128/jvi.02355-13

Wies, E., Wang, M. K., Maharaj, N. P., Chen, K., Zhou, S., Finberg, R. W., et al. (2013). Dephosphorylation of the RNA sensors RIG-I and MDA5 by the phosphatase PP1 is essential for innate immune signaling. Immunity 38, 437-449. doi: 10.1016/j.immuni.2012.11.018

Wu, B., Peisley, A., Richards, C., Yao, H., Zeng, X., Lin, C., et al. (2013). Structural basis for dsRNA recognition. Filament formation, and antiviral signal activation by MDA5. Cell 152, 276-289. doi: 10.1016/j.cell.2012.11.048

Xing, J. J., Wang, S., Lin, R. T., Mossman, K. L., and Zheng, C. F. (2012). Herpes simplex virus 1 tegument protein US11 downmodulates the RLR signaling pathway via direct interaction with RIG-I and MDA-5. J. Virol. 86, 3528-3540. doi: 10.1128/jvi.06713-11

Xing, J., Ni, L., Wang, S., Wang, K., Lin, R., and Zheng, C. (2013). Herpes simplex virus 1-encoded tegument protein VP16 abrogates the production of beta interferon (IFN) by inhibiting NF-kappa B activation and blocking IFN regulatory factor 3 to recruit its coactivator CBP. J. Virol. 87, 9788-9801. doi: 10.1128/jvi.01440-13

Xu, L. G., Wang, Y. Y., Han, K. J., Li, L. Y., Zhai, Z. H., and Shu, H. B. (2005). VISA is an adapter protein required for virus-triggered IFN-beta signaling. Mol. Cell 19, 727-740. doi: 10.1016/j.molcel.2005.08.014

Yamaguchi, T., Kawabata, K., Kouyama, E., Ishii, K. J., Katayama, K., Suzuki, T., et al. (2010). Induction of type I interferon by adenovirus-encoded small RNAs. Proc. Natl. Acad. Sci. U.S.A. 107, 17286-17291. doi: 10.1073/pnas.1009823107

Yang, Z., Xiang, Y. X., Dunker, W., Yu, S., and KarIjolich, J. (2018). RIG-I like receptor sensing of host RNAs facilitates the cell-intrinsic immune response to KSHV infection. Nat. Commun. 9:4841. doi: 10.1038/s41467-018-07314-7

Yoneyama, M., Kikuchi, M., Matsumoto, K., Imaizumi, T., Miyagishi, M., Taira, K., et al. (2005). Shared and unique functions of the DExD/H-box helicases RIG-I, MDA5, and LGP2 in antiviral innate immunity. J. Immunol. 175, 2851-2858. doi: 10.4049/jimmunol.175.5.2851 
Yoneyama, M., Kikuchi, M., Natsukawa, T., Shinobu, N., Imaizumi, T., Miyagishi, M., et al. (2004). The RNA helicase RIG-I has an essential function in doublestranded RNA-induced innate antiviral responses. Nat. Immunol. 5, 730-737. doi: $10.1038 /$ ni1087

Zhang, H. L., Ye, H. Q., Liu, S. Q., Deng, C. L., Li, X. D., Shi, P. Y., et al. (2017). West nile virus NS1 antagonizes interferon beta production by targeting RIG-I and MDA5. J. Virol. 91, e2396-e2416. doi: 10.1128/JVI.02396-16

Zhang, H. R., Ni, G. X., and Damania, B. (2020). ADAR1 facilitates KSHV lytic reactivation by modulating the RLR-Dependent signaling pathway. Cell Rep. 31:107564. doi: 10.1016/j.celrep.2020.107564

Zhang, J., Harnpicharnchai, P., Jakovljevic, J., Tang, L., and Woolford, J. L. (2007). Assembly factors Rpf2 and Rrs1 recruit 5S rRNA and ribosomal proteins rpL5 and rpL11 into nascent ribosomes. Genes Dev. 21, 2580-2592. doi: 10.1101/gad. 1569307

Zhang, J., Wang, K., Wang, S., and Zheng, C. (2013). Herpes simplex virus 1 E3 ubiquitin ligase ICP0 protein inhibits tumor necrosis factor alpha-induced NFkappa B activation by interacting with p65/RelA and p50/NF-kappa B1. J. Virol. 87, 12935-12948. doi: 10.1128/jvi.01952-13

Zhang, X., Wang, C., Schook, L. B., Hawken, R. J., and Rutherford, M. S. (2000). An RNA helicase. RHIV -1, induced by porcine reproductive and respiratory syndrome virus (PRRSV) is mapped on porcine chromosome 10q13. Microb. Pathog. 28, 267-278. doi: 10.1006/mpat.1999.0349

Zhang, Y., Dittmer, D. P., Mieczkowski, P. A., Host, K. M., Fusco, W. G., Duncan, J. A., et al. (2018). RIG-I detects kaposi's sarcoma-associated herpesvirus transcripts in a RNA polymerase III-independent manner. mBio 9, e00823e00918. doi: 10.1128/mBio.00823-18

Zhao, Y., and Karijolich, J. (2019). Know thyself: RIG-I-like receptor sensing of DNA virus infection. J. Virol. 93, e01085-e01119. doi: 10.1128/jvi.01085-19

Zhao, Y., Ye, X., Dunkert, W., Song, Y., and Karijolich, J. (2018). RIG-I like receptor sensing of host RNAs facilitates the cell-intrinsic immune response to KSHV infection. Nat. Commun. 9:4841.
Zhu, H. F., Zheng, C. F., Xing, J. J., Wang, S., Li, S. P., Lin, R. T., et al. (2011). Varicella-zoster virus immediate-early protein ORF61 abrogates the IRF3mediated innate immune response through degradation of activated IRF3. J. Virol. 85, 11079-11089. doi: 10.1128/jvi.05098-11

Zhu, H., and Zheng, C. (2020). The race between host antiviral innate immunity and the immune evasion strategies of herpes simplex virus 1. Microbiol. Mol. Biol. Rev. 84, e00099-e00120. doi: 10.1128/mmbr.0 0099-20

Zuest, R., Cervantes-Barragan, L., Habjan, M., Maier, R., Neuman, B. W., Ziebuhr, J., et al. (2011). Ribose 2'-O-methylation provides a molecular signature for the distinction of self and non-self mRNA dependent on the RNA sensor Mda5. Nat. Immunol. 12, 137-146. doi: 10.1038/ni.1979

Conflict of Interest: JF is employed by Genor Biopharma Co., Ltd.

The remaining authors declare that the research was conducted in the absence of any commercial or financial relationships that could be construed as a potential conflict of interest.

Publisher's Note: All claims expressed in this article are solely those of the authors and do not necessarily represent those of their affiliated organizations, or those of the publisher, the editors and the reviewers. Any product that may be evaluated in this article, or claim that may be made by its manufacturer, is not guaranteed or endorsed by the publisher.

Copyright (c) $2021 \mathrm{Jia}, \mathrm{Fu}$ and Tang. This is an open-access article distributed under the terms of the Creative Commons Attribution License (CC BY). The use, distribution or reproduction in other forums is permitted, provided the original author(s) and the copyright owner(s) are credited and that the original publication in this journal is cited, in accordance with accepted academic practice. No use, distribution or reproduction is permitted which does not comply with these terms. 